\title{
Stairway to Self-Organized Criticality: SOC on a Slope using Relative Critical Heights
}

\author{
Clinton Davis* \\ Department of Physics and Astronomy \\ Ohio Wesleyan University \\ Delaware, Ohio 43015 USA
}

Accepted: February 15, 2008

\begin{abstract}
A computational model of loose snow avalanches was studied as an example of Self-Organized Criticality (SOC). The distribution of the magnitudes of avalanches was measured for a stairstepped system with various critical relative differences and system slopes. Depending on the configuration, the model showed either power law behaviors or a Gaussian distribution. For configurations that displayed power laws, the slope of the power law was dependent on the configuration of the system.
\end{abstract}

\section{INTRODUCTION}

Few people have heard of SelfOrganized Criticality, fewer know what it is, and fewer yet have a good comprehension of it. However, in order to understand the research that will be presented in this article, it is essential that the reader have a basic understanding of the subject. To that end an explanation will now be presented beginning with the individual components: Self-Organization and Criticality.

A self-organized system is a system that tends toward ordered states without the influence of outside controls. If you've ever looked outside after a snowfall you've seen a self-organized system. The snow, if there's no wind, orders itself into a smooth landscape covering any irregularities of the ground under the snow, as seen in Figure 1. The snow flakes have in fact organized themselves into a stable state.

In the context of self-organization, criticality is the tendency of a dynamic system to reach a state past which it must reorganize itself to maintain or increase the stability of the system. In a system of particles, such as snow or sand, this critical point is the moment or state immediately preceding the "avalanche" of particles that

* clinton.davis.2007@gmail.com

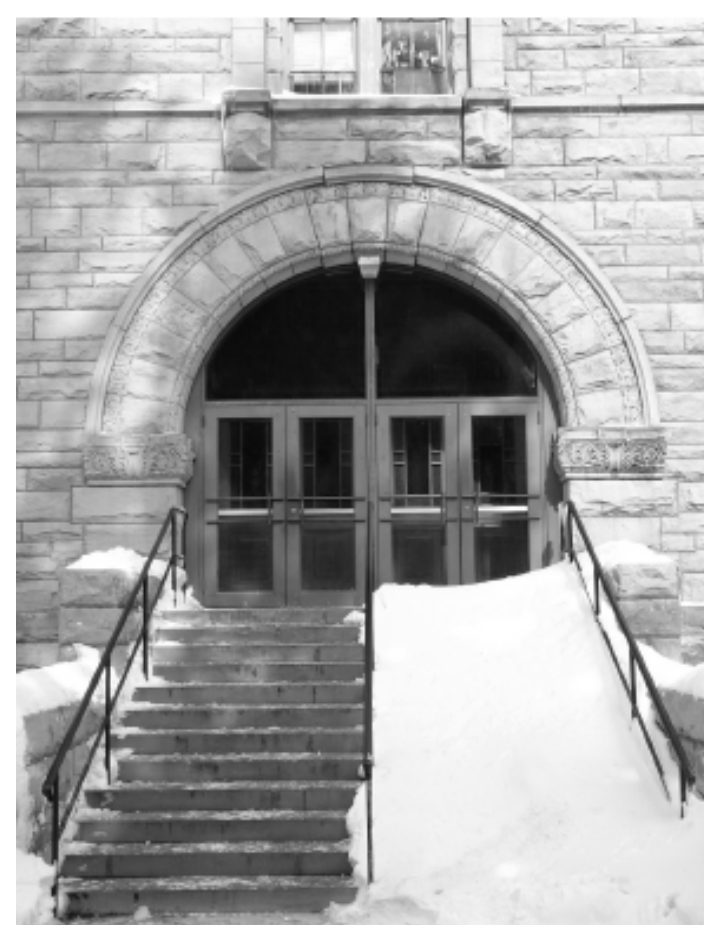

Figure 1. As the snow falls on the stairs of this building, the particles self-organize to smooth the irregularities of the steps.

slide from a configuration of lower stability to a configuration of higher stability, such as from a configuration with a larger difference 

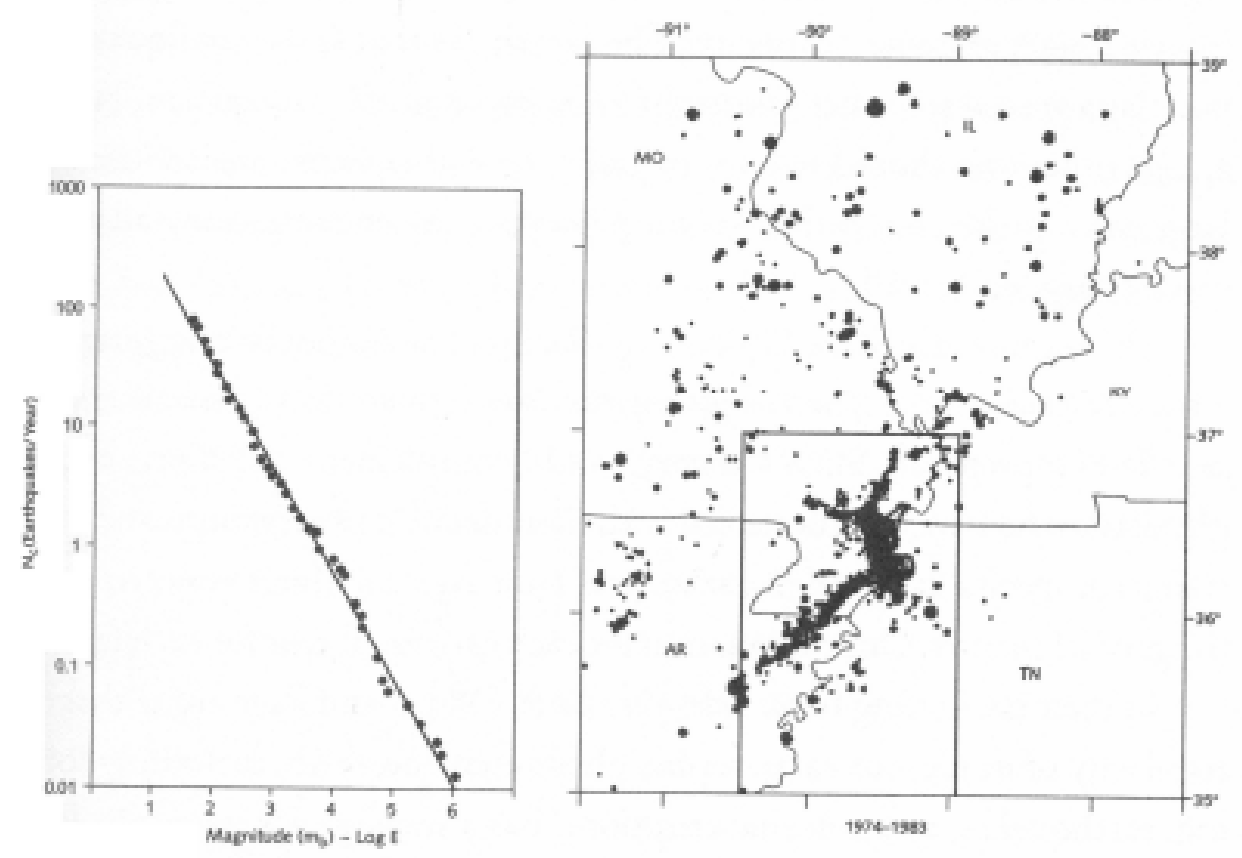

Figure 2. At left is a graph of the Gutenburg-Richter Law in action, plotting data from earthquakes that occurred from 1974-1983 in the New Madrid zone (parts of Arkansas, Illinois, Kentucky, Missouri, and Tennessee). At right is a map of the New Madrid zone showing locations of the earthquakes used in the plot. The sizes of the dots correspond to the magnitudes of the earthquakes [1].

of height between neighboring particles to a configuration with a smaller difference. It is in this way that even if the same amount of snow falls on top of the rock in your yard as the amount that falls next to it, the snow will reorganize itself so that the surface is level, thus causing you to stub your toe as you trudge toward your mailbox.

So, combining these two concepts we find that Self-Organized Criticality (SOC) must be the tendency of a system to tend toward the most stable states possible by reaching critical points followed by avalanches. The avalanches in an SOC system vary in magnitude. Magnitude is defined as the number of toppling events, movements of particles from one location to another that occur in an avalanche. This is where SOC gets interesting. As it turns out, when the logarithm of the magnitude of an SOC avalanche is plotted with the logarithm of the number of avalanches of that magnitude, the graph yields a straight line. This line is representative of a power law, which has the form of $D(s)=a s^{-\tau}$ where $s$ is the magnitude of an avalanche, $D(s)$ is the number (distribution) of avalanches of that magnitude, $-\tau$ is the slope of the linear fit of the log-log plot of $D(s)$ versus $s$, and $a$ is a constant.

An example of a power law is the Gutenburg-Richter Law. It plots the magnitude of an earthquake (which is the log of the energy released in an earthquake) as a function of the distribution of magnitudes as seen in Figure 2 [1].

Zipf [2] looked at similar patterns by ranking cities by population, then plotting rank a function of population. He also looked at the ranks of how often words in the English language were used in various pieces of literature and writing, and compared them to the number of occurrences, shown in Figure 3 . In both circumstances he found a power law relationship.

SOC is interesting for more than just its elegance on a graph or as an excuse for an embarrassing spill in the snow. SOC can also be used as a statistical tool for studying many real world systems that are far too complex to study by individual interactions 


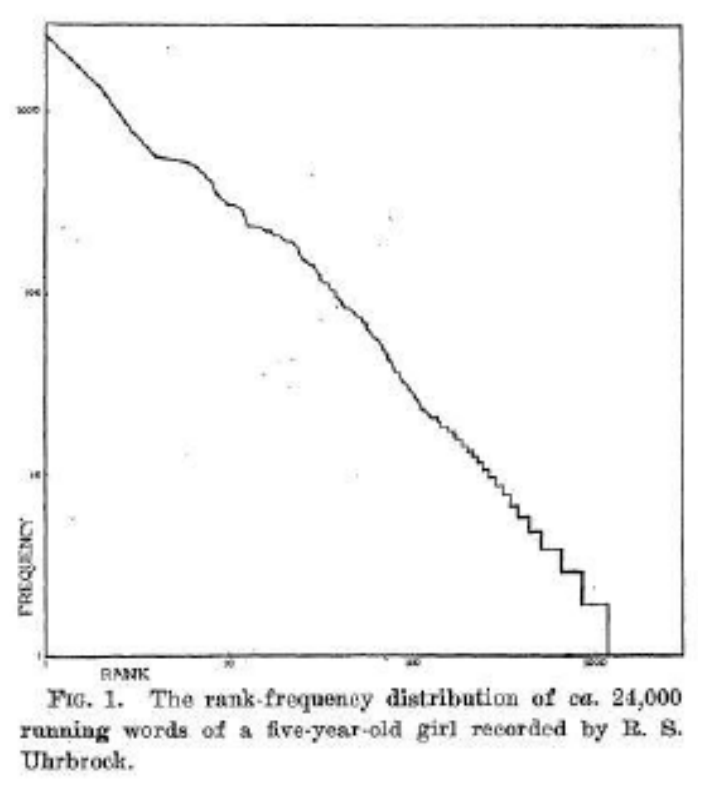

Figure 3. This figure, which appeared in Zipf's article in the October 1942 issue of Science, shows the same type of power law relationship seen in Self-Organized Criticality [2].

and first principles. It can be used to study wild fires, landslides, economic systems, and of course snow slides, all by modeling them with simpler SOC systems.

\section{HISTORY}

\section{a. Origins of Self-Organized Criticality}

SOC was first studied by Per Bak, Chao Tang, and Kurt Wiesenfeld at Brookhaven National Laboratories in the late 1980s [1]. They became interested in $1 / f$ noise, which then led them to the study of what would become SOC. Their goal was to find a simple model that would be both intuitive and general enough to allow meaningful parallels to be drawn to more complex examples. Their first attempt at modeling SOC was a system of many coupled torsion pendulums. This model was "true", but not useful." It gave the correct power law relationship, but was too complex to be helpful in establishing an intuitive understanding of SOC. Bak, Tang and Wiesenfeld then came up with idea of using sand piles as models.

\section{b. Physical Sand Piles}

The physical sand pile model consists of a platform, a computer-controlled sand dispenser, and sand. The sand is added slowly enough to allow careful observation. As the sand accumulates, the pile reaches critical states and then the sand avalanches, causing the pile to grow taller and wider as time progresses until it is as wide as the table, at which point the avalanches carry excess sand off the table and thus out of the system.

Since 1987, several variations on the sand pile have been studied. In one variation instead of the addition of sand causing the stability imbalance necessary to bring about a critical state, a drum partially filled with sand is rotated. This has the effect of increasing the slope of the system until an avalanche occurs (past the critical value of the slope) [3].

Another way that the model has been modified is by replacing sand the sand with larger particles. One model uses glass beads that are all the same size to simulate sand while making the particles large enough to observe individually, which is highly impractical with sand particles. The physics department at The College of Wooster has successfully experimented with various sized glass beads under various constraints [4].

The sand can also be replaced by rice, which is not spherical and has significant lower density and a greater coefficient of friction than glass beads. Because of these factors, it doesn't avalanche as readily and can lead to more complex critical states. The fractal geometry produced in a rice pile (Figure 4) while different from the geometry of other models still follows the power laws that govern SOC.

A third possible variation in the medium of the pile is to use sand of varying wetness. This changes the cohesive properties causing the pile to grow higher before it reaches its critical point. This method is convenient for drawing analogies to snow slides. Snow slides are affected by the wetness of the snow, which is controlled by the temperature. In this analogy, dry sand represents the dry powder snow that occurs under vary cold conditions. Wet sand represents the snow that falls in warmer weather (relatively speaking); the 


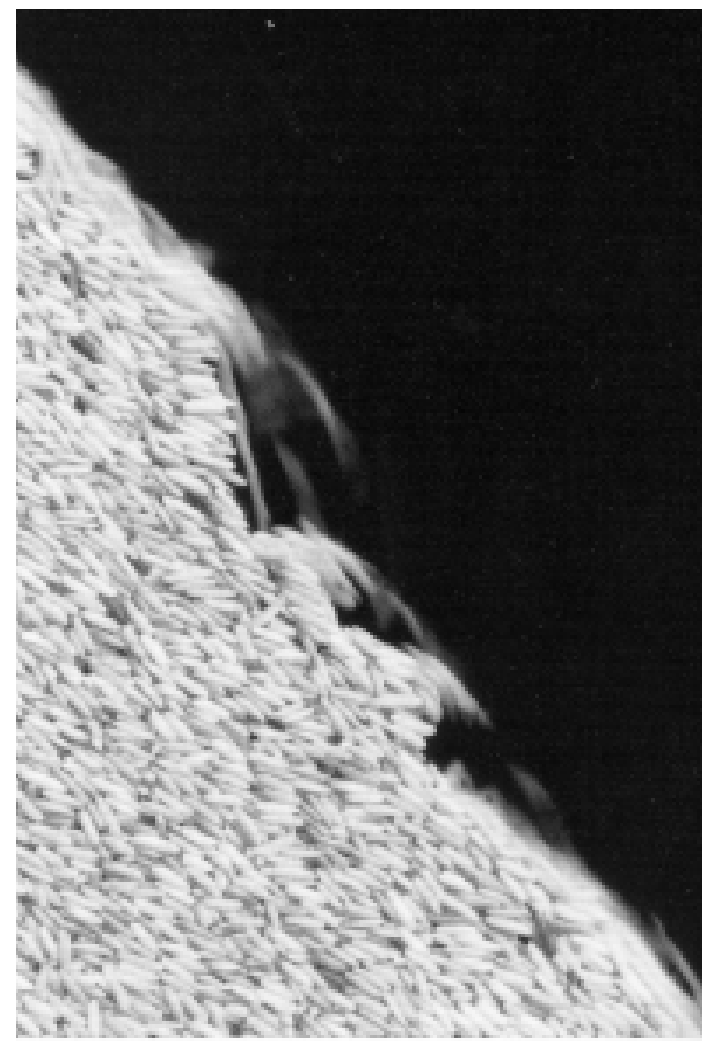

Figure 4. Notice the jagged surface formed as the rice piles. This is a more complex pattern than occurs in piles of sand and spherical beads, which tend to have smooth and regular surfaces.

kind that's good for snowballs and dreadful for skiing.

\section{c. Computer Models of Sand Piles}

As Bak, Tang, and Wiesenfeld discovered, it is often helpful to use computer modeling to simulate a sand pile instead of working with actual physical systems. Through computer modeling, the system can be simplified greatly and data can be taken over a long time without the need for the experimentalist to observe the whole process.

The original Bak-Tang-Wiesenfeld (BTW) model was similar to a checkerboard in which each square was a one particlesized location. Each location was defined by its $x$ and $y$ coordinates; the number of particles it contained was defined as its "height" $z$. If a location reached a height of $z$ $>z_{c}$, that location would pass one of its particles to each of its neighbors, as in Figure 5.
They ran their system two ways. First they started with every location having a height greater than $z_{c}$ and then allowed the system to relax to a stable state which would have some locations with critical heights. Then one of the critical sites was perturbed, that is, forced to pass its particles. This would cause an avalanche ranging in size from one location to the entire system. After the avalanche, the system was reset and the process repeated, They collected data for many avalanches, then plotted avalanche sizes $D(s)$ on a logarithmic graphed. This plot yielded the power law shown in Figure 6.

Second, they started with the heights of every location equal to zero and added particles to random locations one at a time. This allowed the entire system to build up to a critical state at some point, avalanche, and then start building again without resetting. Again they obtained a power law relationship when they plotted $D(s)$ and $s$, as seen in Figure 7. They studied the system with both open and closed boundaries which is equivalent to either having the pile on a table top where the sand can fall off the edges or in a box where it can't, respectively. They also studied the duration of avalanches, $T$, as a function of their distributions, $D(T)$, also obtaining a power law relationship [5].

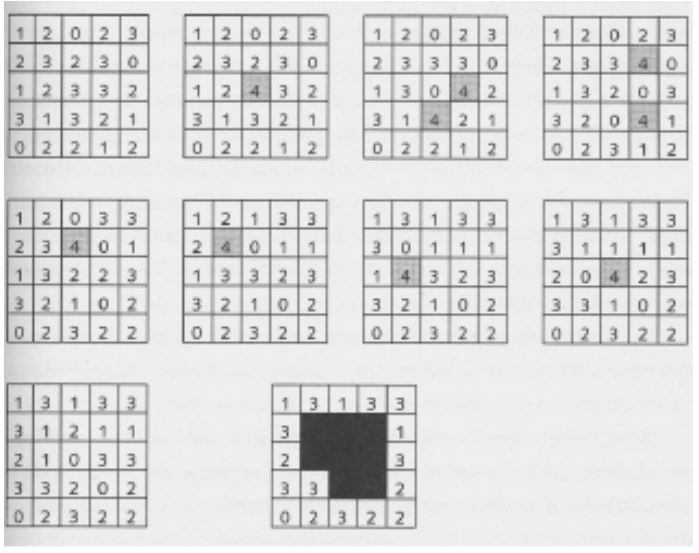

Figure 5. The BTW model for a $5 \times 5$ system with a critical height of $z_{c}=3$. Locations shaded gray are past the critical state. The last picture shows all locations that were involved in an avalanche, of size $s=9$ (meaning that there were 9 toppling events). 


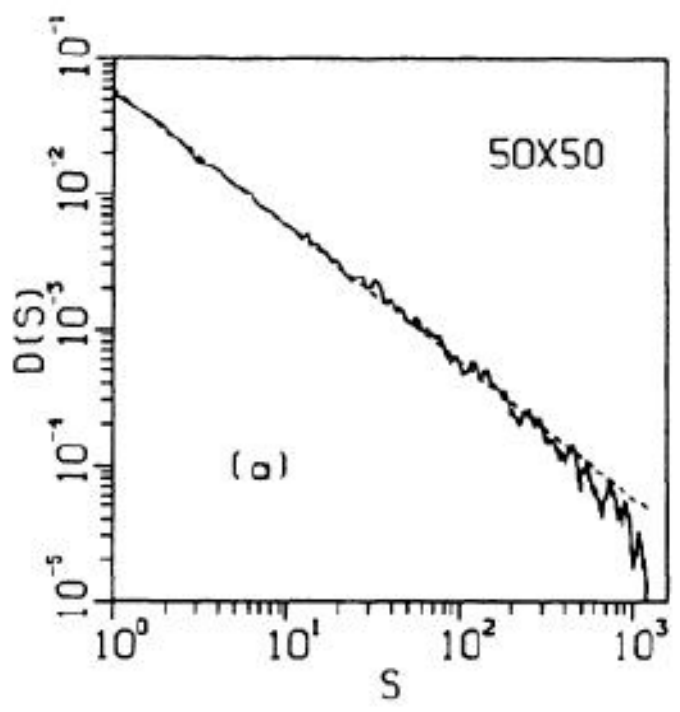

Figure 6. Normalized logarithmic plot of $D(s)$ and $s$ for a $50 \times 50$ BTW model at criticality. The dotted line has a slope of -1 , which means that $\tau$ for this plot is very nearly 1. Data were averaged over 200 samples [5].

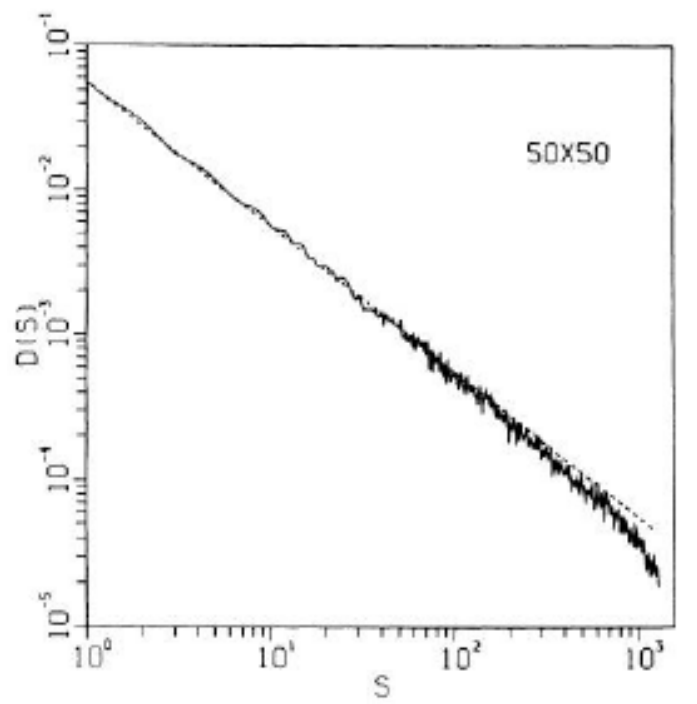

Figure 7. Normalized logarithmic plot of $D(s)$ and $s$ for a $50 \times 50$ BTW model allowed to build from a flat surface. The dotted line has a slope of -1 , which means that $\tau$ for this plot is very nearly 1 . Data were taken for 100,000 iterations. Note the similarity to Figure 6. [5]

Other computer models have been developed including a colorful variant of the BTW model by Michael Creutz [6] (see
Figure 8) that uses different colored pixels to represent locations at various heights. This representation also uses a different color to show locations that are part of an avalanche so that at the end of an avalanche it is very easy to get a qualitative idea of the magnitude of the avalanche [6].

\section{MY RESEARCH MODELS}

\section{a. Loose Snow Models}

Loose snow avalanches are avalanches that begin at a point and form a downhill V shape as they progress (see Figure 9). These slides are in contrast to the often larger and more dangerous slab avalanches which form along a horizontal line and make a rectangular shape on the mountain. Loose snow avalanches make great models for SOC. Each avalanche occurs when a critical slope is reached and continues as long as each new location it reaches is critical as well.

\section{b. First Model}

My first attempt at modeling loose snow avalanches was with a simple onedimensional model resembling a stair step, as seen in Figure 10. Each location, $x$, had an initial integer height of $z(x)$, which was defined by $z_{\text {base }}(x)=x_{\max }-x$, where $x_{\max }$ was the largest $x$ value. A location was considered to be in a critical state when it had a height of $z(x)=k+z(x+1)$, where $z(x+1)$ was the height of the location's downhill neighbor and the integer $k$ was the critical relative height. Particles were added one at a time to a random location until a location exceeded the critical relative height. Upon exceeding the critical relative height, a location would pass one particle to its downhill neighbor. Particles that reached the bottom stair were lost into the void as with a sand pile on a tabletop that loses ant particles that pass the boundary of the edge of the table.

After constructing and studying this system, I found that it was incapable of modeling an SOC system. The problem was that once an avalanche began, it continued through all of the locations below it. Thus, the size of the avalanche was dependent entirely on the starting location, 

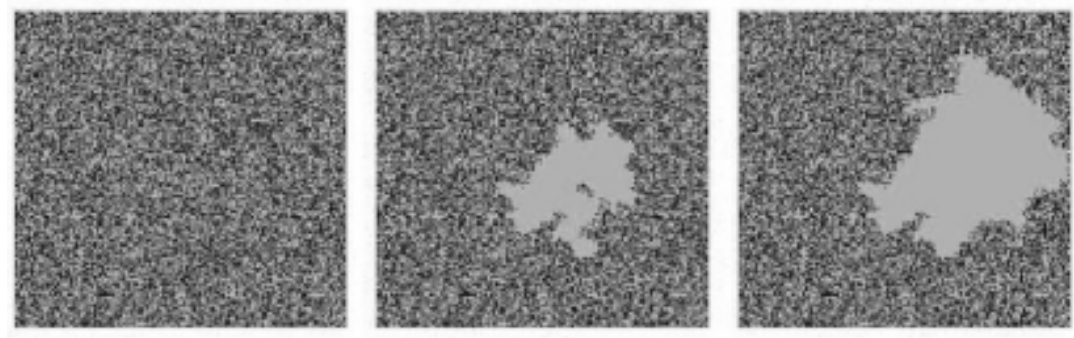

Figure 8. Each color [shown here in grayscale] represents a different height, and each pixel represents a different location in the sand pile. The lighter region shows locations where an avalanche occurred. Like the BTW model, this model has a toppling height of 4. [6]

which does not give the power law relationship of an SOC system.

I attempted to fix this problem by having a critical state pass $(k-1)$ particles, instead of just one particle. This did not solve the problem, so I decided that I would have to rethink my model entirely.

\section{c. Two-Dimensional Model}

The solution to my problem added a whole new dimension to my system. That is, I literally added a dimension to my system. I did this by treating my onedimensional system as a sliver of a twodimensional one, then standing many of them side by side. This created a system that was like that of a checkerboard on a wide set of stairs. Figure 11(a) shows a visual representation of a $15 \times 15$ base system.

The base height for each location was defined to be $z_{\text {base }}(x, y)=\left(x_{\max }-x\right) \cdot g$ where $g$ is the user-defined slope value of the system. In Figure 11, $g=1$. The system was then randomized by adding a random integer number of particles between 0 and $k+1$ to each location so that the system would start out in a critical state, which would then relax during the first iteration. Figure 11(b) shows the randomized system.

In this system, when a location $(x, y)$ surpassed the critical relative height $z(x, y)=$ $k+z(x+1, y)$, instead of only passing its particles to the location directly downhill, $(x+1, y)$. it would also pass to the locations diagonally downhill: $(x+1, y+1)$ and $(x+1, y-1)$. Once again when a particle reaches the lowest step, it leaves the system. This time, however, instead of being walls, the side boundaries are cyclical, that is the locations $(x, 1)$ and $\left(x, y_{\max }\right)$ are neighbors. This allows a smaller system to function like a wider one.

The total number of particles, $q$, to be passed is determined by subtracting the base height of the critical location from its current height:

$$
q=z_{i}(x, y)-z_{\text {base }}(x, y) .
$$

Fractional priority values $\left(P_{\text {Down }}, P_{D L f f t}\right.$, and $\left.P_{\text {DRight }}\right)$ are then determined for each downhill location

$$
((x+1, y),(x+1, y+1),(x+1, y-1))
$$

According to the difference in height between the location and the original (critical) state (see equations (2) - (8)):

$$
\begin{aligned}
& \Delta_{\text {Down }}=z_{i}(x, y)-z(x+1, y) \\
& \Delta_{\text {DLeft }}=z_{i}(x, y)-z_{i}(x+1, y-1) \\
& \Delta_{\text {DRight }}=z_{i}(x, y)-z_{i}(x+1, y+1) \\
& \Delta_{\text {Total }}=\Delta_{\text {Down }}+\Delta_{\text {DLeft }}+\Delta_{\text {DRight }} \\
& P_{\text {Down }}=\frac{\Delta_{\text {Down }}}{\Delta_{\text {Total }}} \\
& P_{\text {Left }}=\frac{\Delta_{\text {Left }}}{\Delta_{\text {Total }}} \\
& P_{\text {Right }}=\frac{\Delta_{\text {Right }}}{\Delta_{\text {Total }}}
\end{aligned}
$$

These fractions are then multiplied by $q$ and the integer parts (see equations (9) - (11) as 


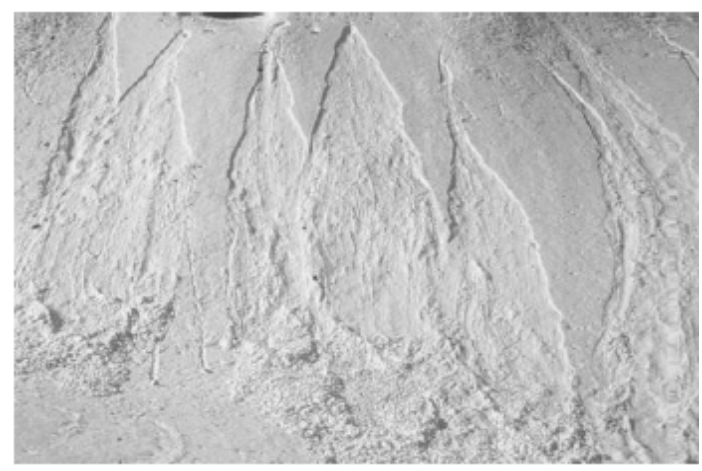

Figure 9. Several loose snow avalanches. Note the inverted $V$ shape [7].

the greatest integer function [ ]) taken to determine the number of particles to be passed to each location:

$$
\begin{gathered}
z_{f}(x+1, y)=z_{i}(x+1, y)+\left[q \cdot P_{\text {Down }}\right] \\
z_{f}(x+1, y-1)=z_{i}(x+1, y-1) \\
+\left[q \cdot P_{\text {DLeft }}\right] \\
z_{f}(x+1, y+1)=z_{i}(x+1, y-1) \\
+\left[q \cdot P_{\text {DRight }}\right]
\end{gathered}
$$

The number of leftover particles, $R$, is then added back to the original location (equations (12) and (13)):

$$
\begin{aligned}
R=q-\left(\left(q \cdot P_{\text {Down }}\right)+\right. & \left(q \cdot P_{\text {DLeft }}\right) \\
& \left.+\left(q \cdot P_{\text {DRight }}\right)\right) \\
z_{f}(x, y)=z_{i}(x, y) & +R
\end{aligned}
$$

If one of the diagonal locations has a height greater than that of the original location, it receives no particles and is not included in the priority calculations.

\section{RESULTS}

I studied my model for various values of $k$ (critical relative difference), $g$ (slope), and the number of iterations. The number of iterations had no qualitative effect on the results: it only changed the scale of the graphs, but not the shapes. The configurations of the parameters $k$ and $g$, however, had significant effects on the results. Some configurations gave the SOC power law relationship that I was looking for; others did not. Figure 12 shows the distribution of magnitudes for various $k$ and $g$ values for a $25 \times 25$ system. There were two classes of results for the various configurations of $k$ and $g$ : a Gaussian and a power law.

\section{a. Gaussian Distribution}

In the region where $(k-g) \leq 1$, the system produced a Gaussian (normal) distribution. Figure 13 shows the Gaussian distribution law that resulted from a system a $k-g$ value of zero.

\section{b. Power Law}

For configurations with $(k-g)>1$ values, the system gave a power law relationship. Figure 14 shows log-log plots of the distribution of avalanches for various values of $k$ and $g$. The exponent, $\tau$, was larger for larger values of $k-g$ and was also larger for larger $g$ (in configurations with equivalent $k-g$ values). All of the power law graphs have some deviation from the power law. This "extra" function becomes less apparent and the data confirm more strictly to the power law for larger values of $(k-g)$. Figure 15 shows the power law that resulted from a system with a relatively large $k-g$ value of 4 .

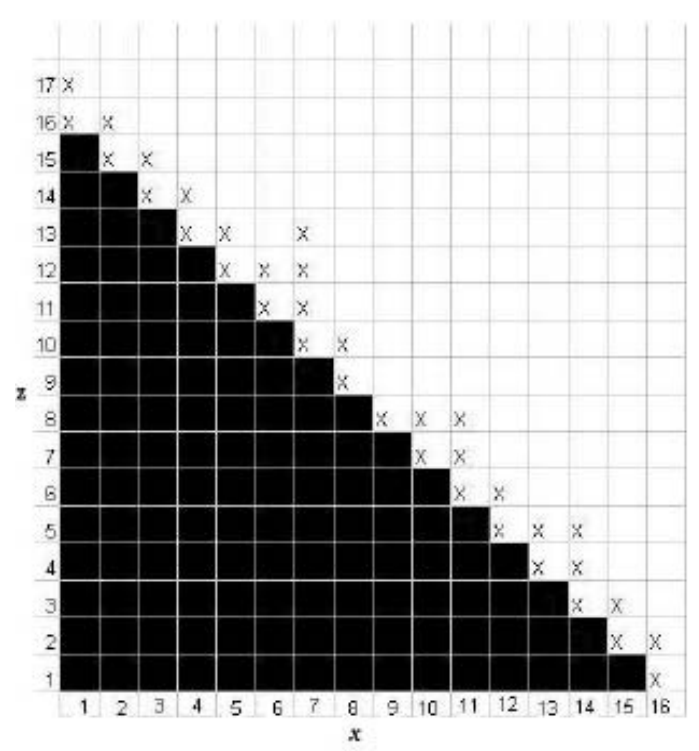

Figure 10. For $k=2, z(7)$ is past critical $[z(7)>k+z(8)]$ and will pass a particle to location $x=8$. (Model was programmed in Mathematica.) 


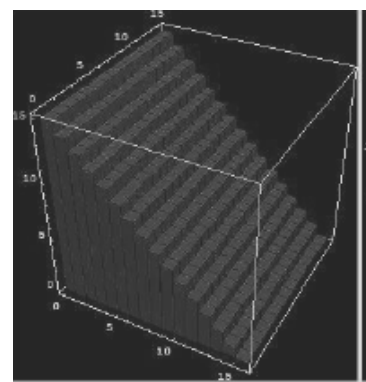

a) Base 2-D Made

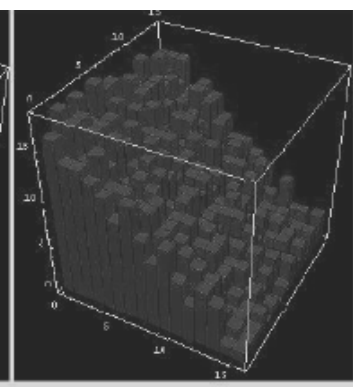

b.) Randomized 2-D Model
Figure 11. My 2-dimensional computational SOC model (programmed in Mathematica).

\section{ANALYSIS}

\section{a. Gaussian Distribution}

The Gaussian distribution of avalanche sizes in the region where $(k-g)$ $\leq 1$ is due to the fact that, as in the one- dimensional system, when a particle is added to a system of that configuration it is very likely it will avalanche all the way through the system. This tendency makes it impossible for the power law to develop. As can be seen in Table 1, there is neither a clearly discernable pattern of change of standard deviation nor of the mean with changes in $k, g$, or $k-g$.

\section{b. Power Law}

The deviations from pure power laws in the systems where $(k-g)>1$ is directly related to the Gaussian distributions discussed in the previous section. currently believe that the deviations are de to the presence of one or more Gaussian terms that inherently underlie the distribution of avalanche magnitudes in my model in

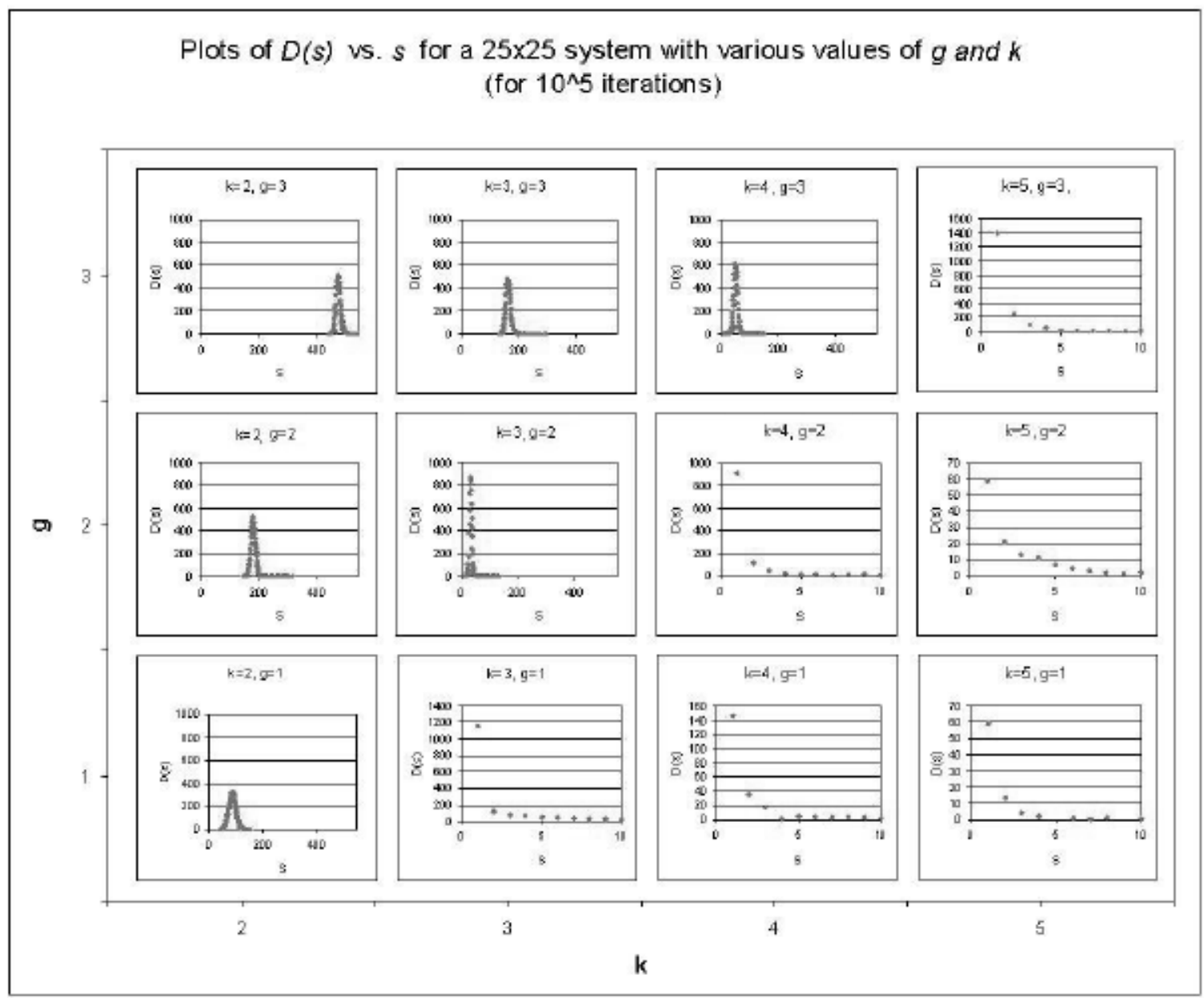

Figure 12. Distribution of avalanche magnitudes for a $25 \times 25$ system, with various $k$ and $g$ values (10 $10^{5}$ iterations). 


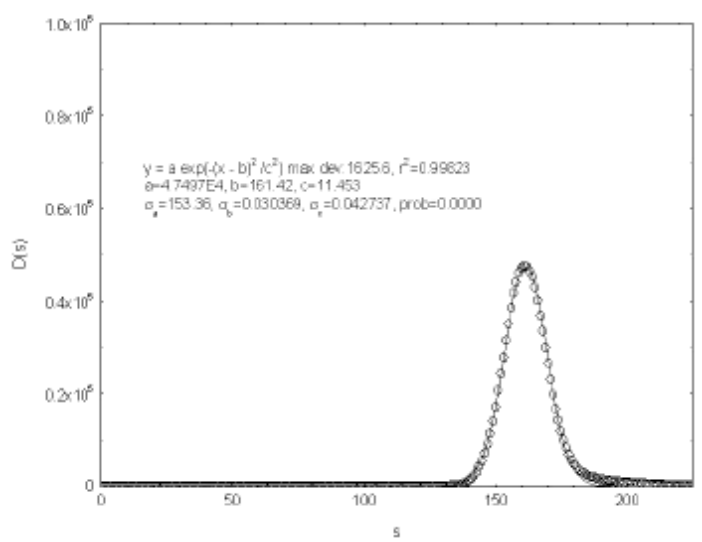

Figure 13. Distribution of avalanche magnitudes for a $25 \times 25$ system with $k=3$ and $g=3\left(10^{6}\right.$ iterations $)$. $(k-g)=0$, Mean $=161.4$, Std. Dev. $=11.5$. addition to the power law term. The deviation is most pronounced near the transition from the Gaussian region to the power law region. For greater values of $(k-$ $g$ ), the power law dominates the graph more, and the presence of the Gaussian is less apparent.

As seen in Table 2, in the power law region $((k-g)>1)$ the quantity $\tau$, the slope of the power law multiplied by -1 , varies with the values of $k, g$, and $k-g$. Most obviously, $\tau$ increases for greater values of $(k-g)$. This indicates that $\tau$ is related inversely to the system's avalanche affinity (how inherently likely the system is to avalanche),

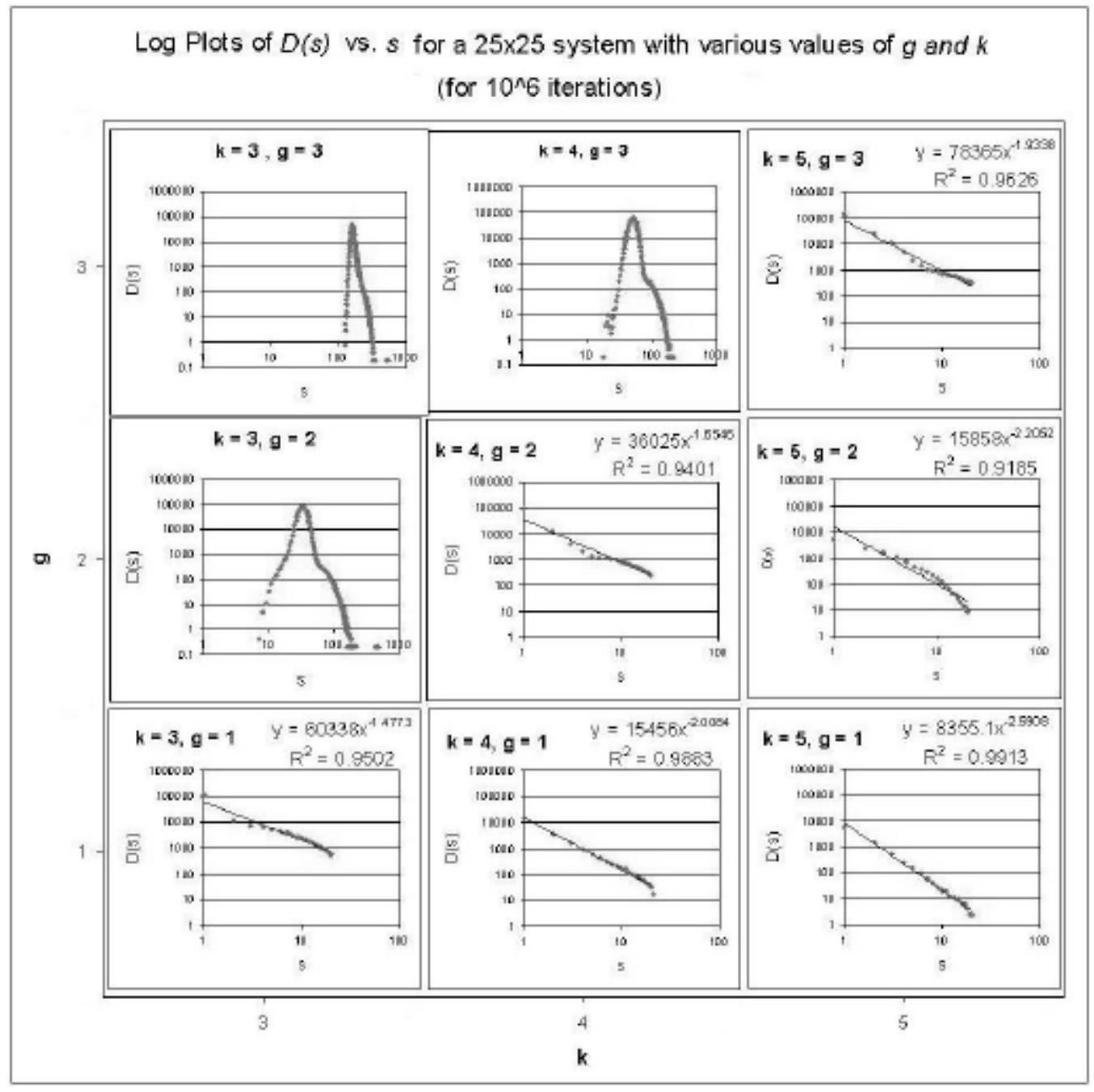

Figure 14. Distribution of avalanche magnitudes for a $25 \times 25$ system with various $k$ and $g$ values on log-log graphs $\left(10^{6}\right.$ iterations). 


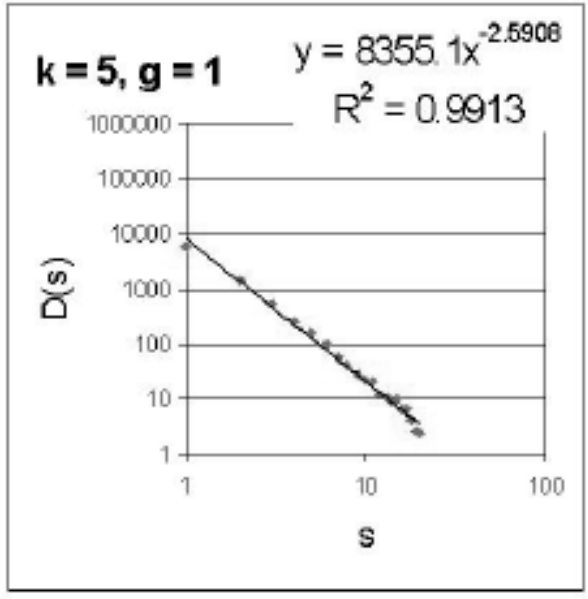

Figure 15. Distribution of avalanche magnitudes for a $25 \times 25$ system with $k=5$ and $g=1$ on log-log axes ( $10^{6}$ iterations) for $\tau=2.5908$.

In addition to the dependence on $(k-g)$, the value of $\tau$ also increases with the values of $k$ and $g$ for configuration with equal values of $k$ $-g$. I do not currently know what causes the dependence on $k$ and $g$ or what significance it has.

\section{CONCLUSIONS AND FUTURE RESEARCH POSSIBILITIES}

Due to the nature of this researchas an undergraduate project and its inherent time restrictions-data sets have been smaller than would be ideal. As a result, our power law data only represents one decade (a span of one order of magnitude) and as such could suffer from finite size effects. Ideally, data would include a least two decades, which would be achieved by running the system for more iterations. However, conclusions will be based on the data collected with the intention of performing longer runs if further research confirms these general results.

Based on the current set of results, I have concluded that this system is an effective model of Self-Organized Criticality when the parameters are chosen so that the noise terms are small $((k-g)>>1)$. I have also concluded that due to its inherent adjustability, this system is also a good model of loose snow avalanches, where $g$ is the slope of the mountainside and $k$ is the wetness/stickiness of the snow. It can then

\begin{tabular}{|c|c|c|c|c|}
\hline $\boldsymbol{k}$ & $\boldsymbol{g}$ & $\boldsymbol{k}-\boldsymbol{g}$ & Mean & $\begin{array}{c}\text { Standard } \\
\text { Dev. }\end{array}$ \\
\hline 2 & 3 & -1 & 16.1 & 11.5 \\
\hline 2 & 2 & 0 & 180.1 & 11.1 \\
\hline 3 & 3 & 0 & 161.4 & 11.5 \\
\hline 2 & 1 & 1 & 86.8 & 18.2 \\
\hline 3 & 2 & 1 & 32.4 & 6.5 \\
\hline 4 & 3 & 1 & 50.1 & 8.7 \\
\hline
\end{tabular}

Table 1. Comparison of $k, g, k-g$, mean, and standard deviation for system configurations in the Gaussian regime $((k-g)$ $\leq 1)$.

\begin{tabular}{|c|c|c|c|}
\hline $\boldsymbol{k}$ & $\boldsymbol{g}$ & $\boldsymbol{k}-\boldsymbol{g}$ & $\boldsymbol{\tau}$ \\
\hline 3 & 1 & 2 & 1.4773 \\
\hline 4 & 2 & 2 & 1.6546 \\
\hline 5 & 3 & 2 & 1.9338 \\
\hline 4 & 1 & 3 & 2.0084 \\
\hline 5 & 2 & 3 & 2.2052 \\
\hline 5 & 1 & 4 & 2.5908 \\
\hline
\end{tabular}

Table 2. Comparison of $k, g, k-g$, and $\tau$ for system configurations in the power law regime $((k-g)>1)$. $\tau$ increases for greater values of $k-g$. It also increases for greater values of $k$ and $g$ in configurations that have the same value of $k-g$.

be inferred that loose snow avalanches in systems with slopes and witnesses correlating to those represented in my system, having values of $(k-g)>1$, should display SOC.

In the future it would be interesting to collect experimental data on loose snow avalanches to test whether they actually can display SOC and to find quantitative relationships between the parameters of my computational system and the parameters of the natural system.

I also look forward to investigating further the deviations from a power law relationship. I have done some preliminary work on attempting to fit a function with both power law and Gaussian terms to the distribution, and have had some promising but preliminary success. I hope to continue in this vein as well as investigate other possible sources for the deviations.

Other interesting possibilities for future research include investigation of the dependence of $\tau$ on $k$ and $g$. For instance, does $\tau$ go to 1 for small values of $g$ as in the BTW model? What are the effects of adding 
obstructions in the system, e.g., randomly altered base heights? What are the effects of changing system sizes, the extension of my system into more dimensions, and the possibility of finding some degree of universality in the results for various configurations?

\section{ACKNOWLEDGEMENTS}

I owe many thanks to Dr. Barbara Andereck for endless help and advising throughout the design, experimentation, and writing of this project; and to Dr. Robert Kaye for his assistance and persistence in the publication of this article. I am also grateful to Drs. Trees, Harmon, and Dillman, Kail Secrest, and the students of the Ohio Wesleyan University Department of Physics and Astronomy for their support, both academic and moral.

\section{REFERENCES}

1. P. Bak, How Nature Works (Copernicus, New York, 1996).

2. G. Zipf, Science, vol. 94 (1942), page 344.

3. G. Baumann and D.E. Wolf, Phys. Rev. E, vol. 54 (1996), page R4504.

4. R.M. Costello, K.L. Cruz, C. Egnatuk, D.T. Jacobs, M.C. Krivos, T.S. Louis, R.J. Urban, and H. Wagner, Phys. Rev. $E$, vol. 67 (2003), page 041304.

5. P. Bak, C. Tang, and K. Wiesenfeld, Phys. Rev. A, vol. 38 (1988), page 364.

6. M. Creutz, Physica A, vol. 340 (2004), page 521.

7. W.G.E.A.F. Services, Glossary Snow and Avalanches (2007). URL http://wa/slf.ch/index. php?id=278.

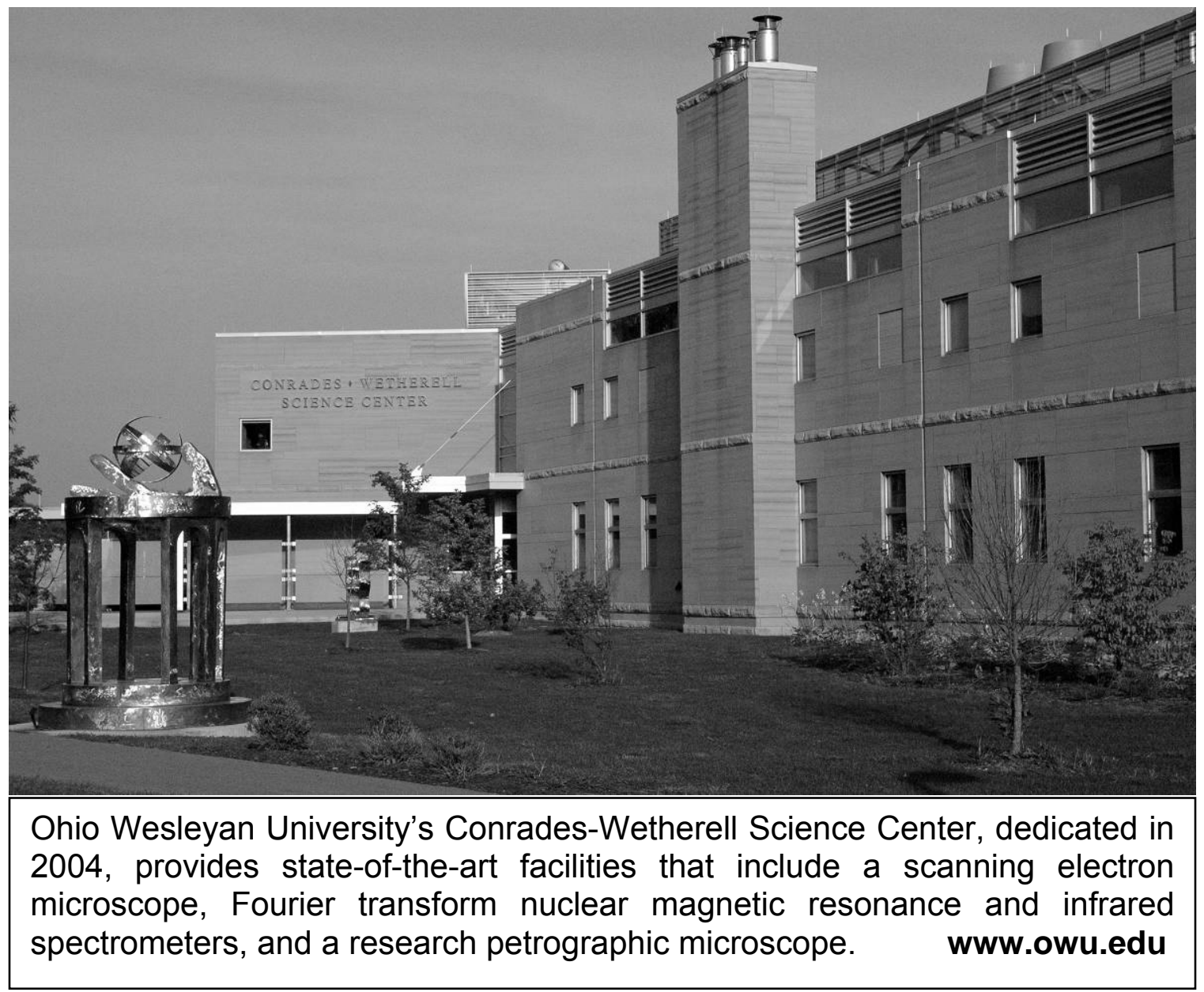




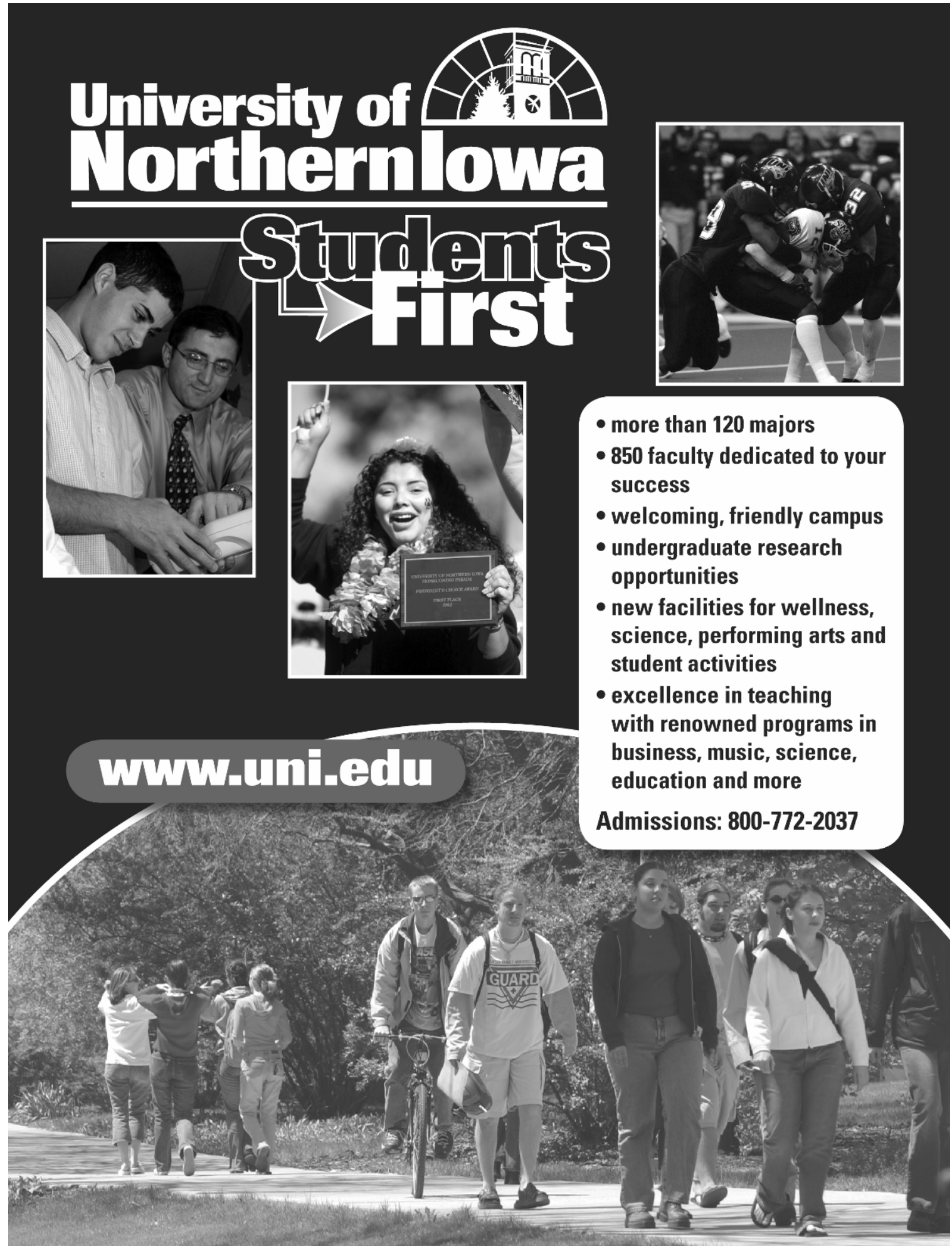

